\title{
Sobrepeso y obesidad como precursores de gingivitis.
}

\section{Overweight and obesity as a precursor to gingivitis.}

\author{
Ramos-Siguencia Jorge*1, Flores-Regalado Carol $^{2}$, Vélez-Bravo Paúl ${ }^{2}$, Sánchez-Zamora Vilmo René ${ }^{3}$ \\ ${ }^{1}$ Odontólogo de práctica privada ${ }^{2}$ Odontólogo en servicio rural ${ }^{3}$ Máster en Ciencias, Docente de la Carrera de Odontología \\ de la Universidad Católica de Cuenca-Ecuador \\ * jeramoss77@gmail.com
}

\begin{abstract}
Resumen
Objetivos: Determinar la relación de la obesidad con la gingivitis utilizando el Índice de Masa Corporal (IMC) como indicador de obesidad y los indicadores de salud bucal como guía de la salud gingival. Establecer la relación entre la presencia de placa bacteriana y sujetos obesos. Materiales y métodos: El presente estudio tiene un nivel de investigación relacional con un corte de temporalidad retrospectivo. De 1600 fichas clínicas tomadas de la base digital de preconsulta de la clínica odontológica de la Universidad Católica de Cuenca, período septiembre 2018 - enero 2019; se tomó una muestra de 352 fichas utilizando criterios de inclusión y exclusión para su respectivo análisis en el apartado del IMC y el indicador de salud bucal de cada paciente. Resultados: La curva en los puntos de dispersión para los pacientes con gingivitis reflejó un IMC más alto en comparación a lo proyectado en otros grupos de estudio. Los resultados obtenidos fueron 0,049 en la escala de R Pearson y 0,066 en la $R$ Spearman. Conclusión: Observamos un patrón de crecimiento de la curva directamente proporcional a la relación: sobrepeso, obesidad - gingivitis. No existe relación directa entre la acumulación de placa bateriana con pacientes obesos, en virtud de estos resultados podemos decir que los procesos metabólicos son desencadenantes de estados de gingivitis en las personas obesas más no la acumulación de placa. Sin embargo, se encontró una correlación baja en cuanto estas variables.
\end{abstract}

Palabras clave: Índice de Masa Corporal, Sobrepeso, Obesidad, Gingivitis, Placa Dental.

\begin{abstract}
Objectives: Determine the relationship between obesity and gingivitis using Body Mass Index (BMI) as an indicator of obesity and oral health indicators as a guide to gingival health. Determine he relationship between the presence of bacterial plaque and obese subjects. Materials and methods: The present study has a relational research level with a retrospective temporal cut. From 1600 clinical cards taken from the digital base of pre-consultation of the dental clinic of the Universidad Católica de Cuenca, period September 2018 - January 2019; a sample of 352 cards using inclusion and exclusion criteria for their respective analysis in the Body Mass Index section and the oral health indicator of each patient. Results: The curve in the dispersion points for patients with gingivitis reflected a higher BMI compared to that projected in other study groups. The results obtained were 0.049 on the R Pearson scale and 0.066 on the R Spearman scale. Conclusion: We observe a pattern of growth of the curve, directly proportional to the relationship: overweight, obesity - gingivitis. There is no direct relationship between the accumulation of baterial plaque in obese patients; by virtue of these results, it indicates that metabolic processes are triggers in states of gingivitis in obese people, and not the accumulation of plaque. However, no statistically significant differences were found.
\end{abstract}

Key words: Body Mass Index, Overweight, Obesity Gingivitis, Dental Plaque.

\section{INTRODUCCIÓN}

La obesidad es una de las enfermedades no transmisibles más prevalentes en la población mundial, actualmente un tercio de esta la padece. ${ }^{1}$ Se trata de una patología compleja y multifactorial que resulta del almacenamiento excesivo de grasa, donde actúan factores sociales, conductuales, culturales, psicológicos, metabólicos y genéticos. ${ }^{2}$ En 2016 más de 1900 millones de adultos tenían sobrepeso; de los cuales más de 650 millones eran obesos. ${ }^{1,3}$
Según la última encuesta de salud y nutrición (ENSANUT) periodo 2011-2013, llevada a cabo por el Instituto Nacional de Estadística y Censos (INEC); en Ecuador la prevalencia de sobrepeso y obesidad en adultos de 20 a 60 años fue de 4'876.076 (62.8\%). Por otra parte, los adultos mayores de 60 años con sobrepeso y obesidad son 682.109 (59.0\%), en general el país tiene 5'558.185 de personas que padecen esta condición. ${ }^{4}$

Se considera la obesidad como factor de comorbilidad 


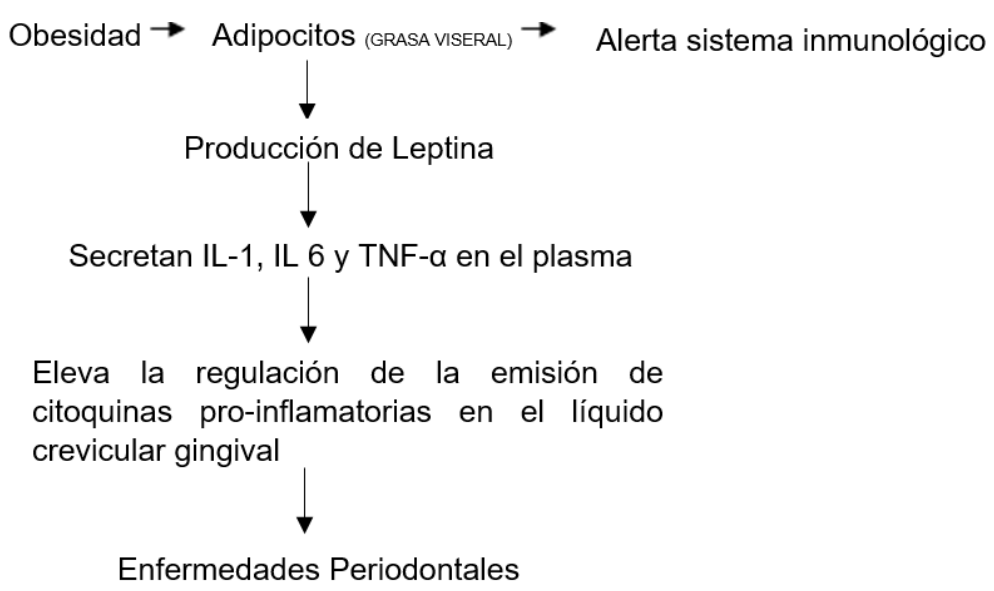

Fig. 1. Proceso de inflamación causado por la relación obesidad y gingivitis.

para adquirir enfermedades como: diabetes tipo II, hipertensión, hipoventilación, apnea obstructiva del sueño, estasis venosa, cáncer, enfermedades degenerativas de las articulaciones, entre otras. ${ }^{2,5}$

Las enfermedades periodontales son un conjunto de patologías que afectan a los tejidos de soporte y protección de las piezas dentarias, son producidas principalmente por bacterias anaerobias gram-negativas. ${ }^{6}$ Según la última clasificación vigente, las enfermedades periodontales son claramente diferenciables tanto clínica como histológicamente, se dividen en dos categorías principales: gingivitis y periodontitis. ${ }^{7}$

La gingivitis puede estar o no inducida por placa bacteriana. La primera, se define como una inflamación local provocada por el acúmulo de biofilm dentro de la bolsa gingival, que normalmente no se extiende hasta la inserción periodontal. $^{7}$

Las alteraciones gingivales no inducidas por placa son un grupo de trastornos que no se resuelven tras la eliminación de la placa. Estas lesiones pueden estar localizadas en los tejidos gingivales o ser manifestaciones de ciertas enfermedades sistémicas. ${ }^{8}$ Aún así, hay que recordar que la gravedad de estos trastornos puede aumentar por el acúmulo de placa. ${ }^{9}$

La característica principal de la periodontitis es la pérdida de soporte de los tejidos periodontales debida a inflamación, habitualmente se utiliza como umbral la pérdida de inserción clínica (PI) interdentaria en dos o más dientes no adyacentes, o bien PI vestibular $\geq 3 \mathrm{~mm}$ con bolsas más de $3 \mathrm{~mm}$ en dos o más dientes. ${ }^{7}$

Varios estudios epidemiológicos relacionan la enfermedad periodontal con la obesidad, sugiriendo otro factor etiológico para la patología. Al tener efectos en la salud sistémica, modifican la susceptibilidad del huésped debido a la producción de mediadores inflamatorios. ${ }^{2,5}$

La posible interacción entre la obesidad y las enfermedades periodontales se basa en el aumento de los niveles de adipocitos, éstas sustancias tienen un rol fundamental en la respuesta inmunitaria del huésped. Mientras mayor sea la cantidad de tejido adiposo mayores son los niveles de leptina interleucina IL-1 e IL-6, del factor de necrosis tumoral (TNF)- $\alpha$; aumentando los niveles de citoquinas inflamatorias en el líquido crevicular gingival, lo que acelera el progreso de la inflamacion periodontal. ${ }^{12}$ Estos factores están involucrados en las patofisiologías proinflamatorias a nivel sistémico de los sujetos obesos. ${ }^{10,11}$ De este modo el IMC elevado y los mediadores inflamatorios; en conjunto, incrementan la susceptibilidad a padecer enfermedades periodontales. $^{2,13}$ (Figura 1.)

Se plantearon dos objetivos en el presente estudio, determinar la relación de la obesidad con la gingivitis utilizando el Índice de Masa Corporal como indicador de obesidad y los indicadores de salud bucal como guía de la salud gingival; además de establecer la relación entre la presencia de placa bacteriana y sujetos obesos, en pacientes que acuden al área de pre-consulta médica en la Carrera de Odontología de la Universidad Católica de Cuenca.

Considerando la tendencia creciente de la obesidad y el hecho de que el aumento del IMC es un importante factor de riesgo para la aparición de la enfermedad periodontal, en este estudio consideramos importante evaluar la relación entre el IMC y la gingivitis.

Como profesionales de la salud, los odontólogos 
debemos comprender la importancia del manejo de pacientes de riesgo metabólico. Para tal efecto debe efectuarse monitoreo periódico en pacientes con sobrepeso y obesidad, así contribuimos al diagnóstico temprano de enfermedades periodontales y ampliamos el criterio clínico del odontólogo.

\section{MATERIALES Y MÉTODOS}

El presente estudio tiene un nivel de investigación relacional con un corte de temporalidad retrospectivo. De 1600 fichas clínicas tomadas de la base digital de preconsulta de la clínica odontológica de la Universidad Católica de Cuenca, período septiembre 2018 - enero 2019; se tomó una muestra de 352 fichas utilizando criterios de inclusión y exclusión para su respectivo análisis en el apartado del Índice de Masa Corporal y el indicador de salud bucal de cada paciente.

Las variables de inclusión del presente estudio fueron: fichas de sujetos sistémicamente sanos, fichas correctamente llenadas que incluyen peso, altura y edad documentados, sexo, historia clínica, e índice de salud bucal correctamente llenado. Los criterios de exclusión constan: pacientes edéntulos, sujetos que tomen medicamentos antinflamatorios por sus enfermedades crónicas, pacientes que estén bajo un tratamiento periodontal, mujeres embarazadas y madres lactantes.

Utilizando la clasificación internacional recomendada por la Organizacion Mundial de la Salud, categorizamos a los pacientes del siguiente modo: bajo peso-peso normal $\leq 18,50$ - 24,99 kg/m2, sobrepeso $25,00-29,99 \mathrm{~kg} / \mathrm{m} 2$ y obesidad I-II-III $\geq 30,00 \mathrm{~kg} / \mathrm{m} 2$. $^{5}$ Se juntaron las categorías de bajo peso-peso normal y obesidad tipo I, II, III, en una sola clasificación para fines estadísticos.

El índice de salud bucal fue evaluado siguendo criterios del manual de uso de los formularios básicos de la historia clínica única 001, emitida por el Ministerio de Salud Pública del Ecuador en base a la cual nos regimos. ${ }^{14}$

Los indicadores de salud bucal analizados fueron el de placa dental y gingivitis omitiendo el de cálculo dental. Analizamos las 6 piezas dentales registradas con el número de pieza correspondiente, estas debían estar completamente erupcionadas el momento de realizar el exámen

En la Columna Piezas Dentales los tratantes marcan con una X la pieza dental presente en boca por examinar. Se continúa en la misma fila para registrar en números en las columnas contiguas de placa y gingivitis. El grado de placa se registra por $0=$ ausencia de placa, $1=$ placa a nivel del tercio gingival, $2=$ placa hasta tercio medio, $3=$ placa en toda la superficie, y en la gingivitis, $0=$ ausencia de sangrado y $1=$ presencia de sangrado. De estos dos cuadros, se suma el total, luego divide por el número de piezas analizadas y se saca un promedio general de cada columna.

\begin{tabular}{|c|c|c|c|c|c|c|c|}
\hline \multicolumn{8}{|c|}{7 INDICADORES DE SALUD BUCAL } \\
\hline \multicolumn{8}{|c|}{ HIGIENE ORAL SIMPLIFICADA } \\
\hline \multicolumn{5}{|c|}{ PIEZAS DENTALES } & \multirow{2}{*}{$\begin{array}{c}\text { PLACA } \\
0-1-2-3 \\
1\end{array}$} & \multirow{2}{*}{$\begin{array}{c}\text { CÁlCULO } \\
0-1-2-3 \\
0\end{array}$} & \multirow{2}{*}{$\begin{array}{c}\text { GINGIVITIS } \\
0-1 \\
1\end{array}$} \\
\hline 16 & $\otimes$ & 17 & & 55 & & & \\
\hline 11 & $\otimes$ & 21 & & 51 & 1 & 0 & 1 \\
\hline 26 & & 27 & Q & 65 & 1 & 1 & 1 \\
\hline 36 & & 37 & Q) & 75 & 0 & 0 & 0 \\
\hline 31 & $\otimes$ & 41 & & 71 & 1 & 0 & 0 \\
\hline 46 & Q & 47 & & 85 & 1 & 1 & \\
\hline \multicolumn{5}{|c|}{ TOTALES } & 0,83 & 0,33 & 0,5 \\
\hline
\end{tabular}

Fig. 2. Indicadores de Salud Bucal

Los datos fueron analizados usando el programa estadístico IBM SPSS Statistics 25, se empleó el coeficiente de correlación de Spearman y Pearson para determinar la relación del IMC con la gingivitis y placa bacteriana.

\section{RESULTADOS}

Un total de 352 fichas clínicas de pacientes de la clínica Odontologíca de la Universidad Católica de Cuenca se incluyeron para la investigación, de este total 207 pacientes fueron de sexo femenino $(58,8 \%)$ y $145(41,2 \%)$ de sexo masculino. Las edades comprendidas en el grupo de estudio fueron de 15 a 66 años de edad, con un promedio de 36,8 años.

En cuanto a los resultados de la variable IMC los promedios encontrados fueron $112(31,8 \%)$ sujetos obesos clase I, II, III; 119 (33,8 \%) con sobrepeso; 120 (34,4\%)bajo peso y peso normal.

Observamos una relación no lineal entre las variables gingivitis y el IMC en cuanto al sexo de los participantes, apreciándose un aumento de la curva que muestra un patrón creciente de la gingivitis conforme va aumentado el IMC. Además, no se encontró una diferencia en el sexo masculino y femenino respecto a la gravedad con la presencia de gingivitis. (Gráficos 1-2) 


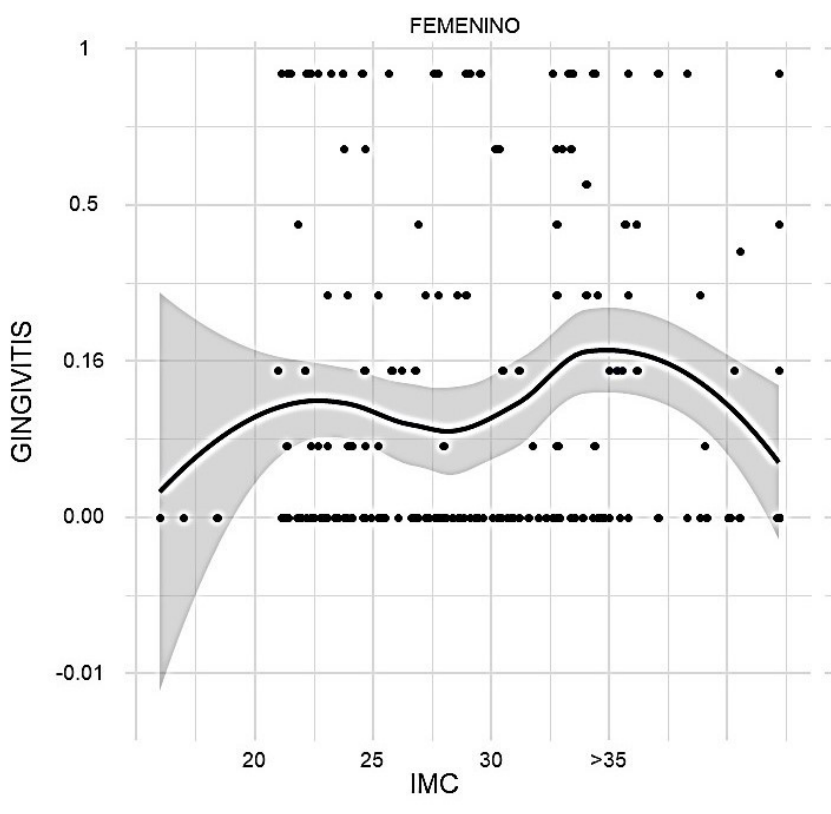

Grf. 1. Puntos de dispersión en la relación al Índice de Masa Corporal (IMC) y gingivitis en el grupo de sexo Femenino

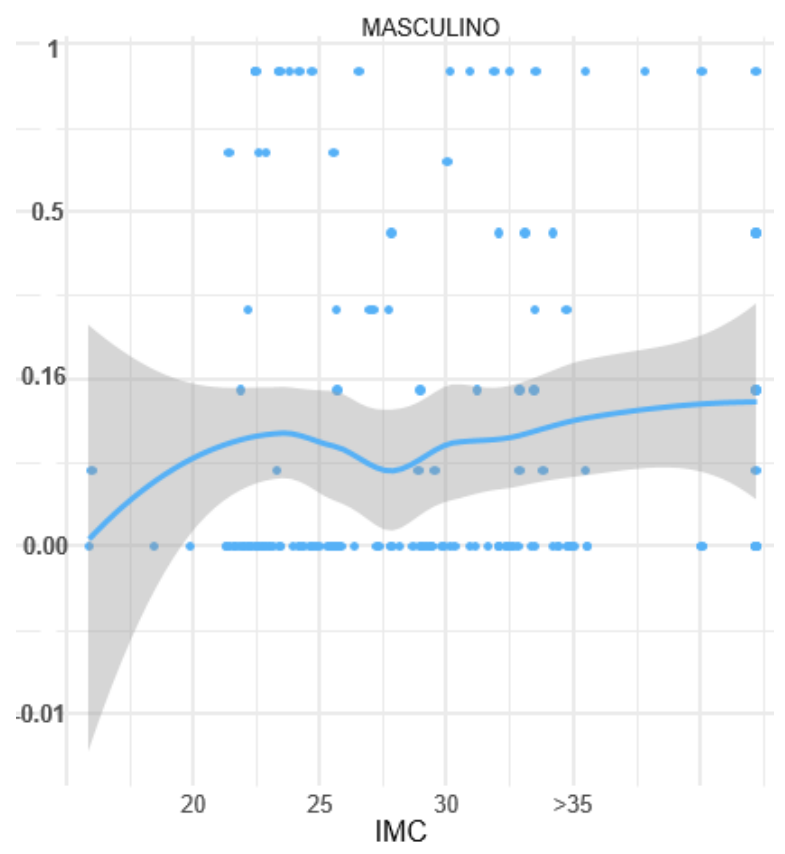

Grf. 2. Puntos de dispersión en la relación al Índice de Masa Corporal (IMC) y gingivitis en el grupo de sexo Masculino

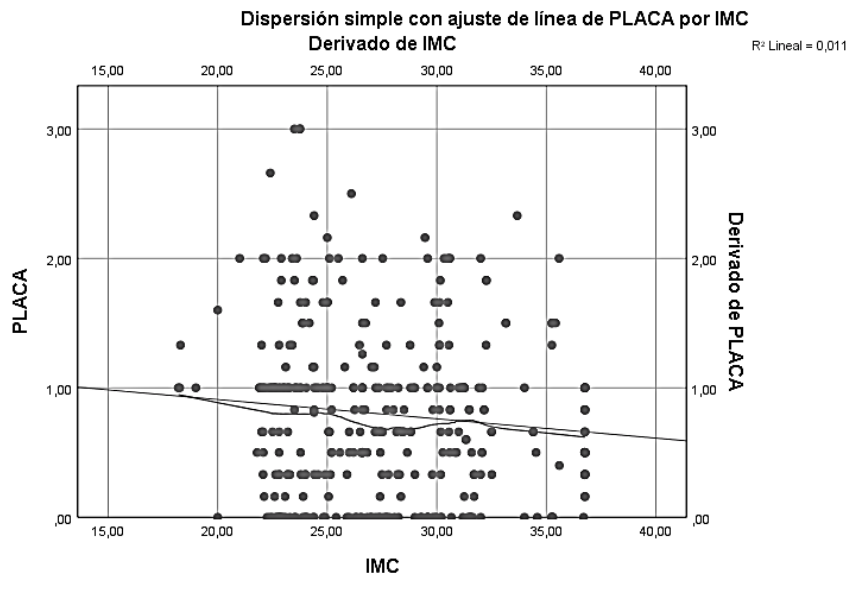

Grf. 3. Puntos de dispersión en la relación al Índice de Masa Corporal (IMC) y Placa dental.

Observamos una curva descendente en los puntos de dispersión. A medida que aumenta el IMC, disminuye la cantidad de placa dental acumulada. (Grf. 3)

Observamos una correlación baja en cuanto el IMCgingivitis e IMC-placa bacteriana. Aceptando la hipótesis nula. (Tabla 1.)

Tabla 1. Tabla de correlación de Pearson y Spearman

\begin{tabular}{ccc}
\hline & R de Pearson & Spearman \\
\hline IMC-Gingivitis & $\mathbf{0 , 0 4 9}$ & $\mathbf{0 , 1 0 3}$ \\
IMC-Placa & 0,066 & 0,092 \\
\hline
\end{tabular}

\section{DISCUSIÓN}

Los resultados de la investigación reflejan que pacientes con un IMC mayor a $25 \mathrm{~kg} / \mathrm{m} 2$ son suceptibles a presentar gingivitis. Este hallazgo concuerda con estudios previos, como el de Ansari S, ${ }^{15}$ quien reporta en su investigación que sujetos con gingivitis tenían un IMC elevado ( $\geq 25 \mathrm{~kg} / \mathrm{m} 2)$, que los sujetos con IMC normal (18,50 - 24,99 kg/m2).

La correlación de sobrepeso y obesidad con la gingivitis es muy baja en nuestro estudio (RP 0,049), similar a lo reportado por Zuza et al, ${ }^{17}$ quienes encontraron una relación leve entre obesidad-gingivitis (RP 0,61). Por el contrario Muniz et $a l,{ }^{16}$ observaron un valor significativo para la relación obesidad-gingivitis (RP 1.22) y moderado para sobrepeso-gingivitis (RP 0,58).

En el estudio de Martinez et $a{ }^{18}{ }^{18}$ donde citan datos epidemiológicos sobre la asociación entre obesidad y enfermedad periodontal; observamos que en Korea, Finlandia y Dinamarca no existe relación significativa 
entre estas variables; mientras que en Reino Unido y Estados Unidos si demuestran la relación. Nuestro estudio realizado en Ecuador no encontró relación estadísticamente significativa.

El hecho de haber obtenido valores bajos en nuestro estudio lo podemos atribuir a lo manifestado por Chopra et $a{ }^{20}$ quienes concluyen que la obesidad sólo se asociará con la enfermedad periodontal si es definida por la circunferencia de la cintura (varones $\geq 102 \mathrm{~cm}$ - mujeres $\geq 88 \mathrm{~cm}$ ) y no necesariamente cuando se mide con el IMC. Pues la distribución anatómica de los depósitos de tejido adiposo determinará que la distrbución central es más activo metabólicamente que el periférico.

En cuanto a la relación IMC con la cantidad de placa bacteriana, observamos que conforme aumenta el IMC, disminuye la acumulación de depósitos blandos. De igual manera Franchini y otros, ${ }^{21}$ indican que la gingivitis en individuos con IMC elevado se debía muy probablemente a la combinación de sus perfiles metabólicos e inflamatorios, más no a una higiene bucal deficiente. Contrastando estos argumentos, el estudio de Fadel et al, ${ }^{22}$ mencionan que la mayoría de los sujetos obesos presentaban gingivitis por malos hábitos de higiene bucal.

Israel Juárez et al, ${ }^{23}$ aportan que considerar la obesidad abdominal como factor de riesgo para la gingivitis y las enfermedades periodontales, obligaría a cambiar el enfoque terapeútico para los pacientes obesos. No sólo requerirán atención para su condición sistémica; sino también control y mantenimiento periodontal, ya que su salud oral se vería más afectada que la de los pacientes no obesos.

\section{CONCLUSIÓN}

Se observó una tendencia positiva entre sobrepeso, obesidad, gingivitis. Sin embargo la correlación no fue estadisticamente significativa.

No existe relación directa entre la presencia de mayor 10 cantidad de placa bacteriana con el IMC elevado; en virtud de estos resultados, indica que los procesos metabólicos son desencadenantes en el desarrollo de inflamación en tejidos gingivales en personas obesas, más no la acumulación de placa bacteriana. Se encontró una correlación baja en cuanto estas variables.

\section{CONFLICTO DE INTERESES}

Los autores no manifiesta ningún conflicto de interés.

\section{AGRADECIMIENTOS}

Los Autores agradecen a:

- Dr. Diego E. Palacios Vivar (Departamento de
Investigación, Carrera de Odontología, Universidad Católica de Cuenca) por su apoyo en la edición y la revisión crítica.

- Dr. Ebingen Villavicencio Caparó (Departamento de Investigación, Carrera de Odontología, Universidad Católica de Cuenca) por su apoyo en la edición y la revisión crítica.

- Dr. Stefano Torracchi Carrasco (Departamento de Investigación, Carrera de Odontología, Universidad Católica de Cuenca) por su ayuda en la parte estadística.

\section{REFERENCIAS BIBLIOGRÁFICAS}

1 Organización Mundial de la Salud. Obesidad y Sobrepeso. Ginebra, Suiza: OMS; 2016; p. 1-18

2 Crispino A, Valentina L. Obesity and periodontal disease. Acta Biomedica Scientia. 2016 Oct; 3(1): p. 6-8.

3 Giri, Dhirendra The relationship of Obesity and Periodontal disease among Urban Population of Biratnagar, Nepal. Orthodontic Journal of Nepal. 2018 Jun; 8(1): p. 45-47.

4 Freire WB, Ramírez MJ, Belmont P, Silva MK, Romero N, Sáenz K, Piñeiros P, Gómez LF, Monge R. Resumen ejecutivo. TOMO I. Encuesta Nacional de Salud y Nutrición del Ecuador. ENSANUT-ECU 2011-2013 Ministerio de Salud Pública/Instituto Nacional de Estadística y Censos. Quito, Ecuador. 2013 1(1): p 57

5 Kumar A, Kumar M, Rao K, Dileep K. Periodontal disease and obesity. International Journak of stomatology y occlusion medicine. 2013 Jun; 6(1): p. 1-5.

6 Correa M, Rengifo C. Rev. Clin. Periodoncia Implantol. Rehabil. 2013; Oral Vol. 6(3): p. 142-147.

7 Chapple ILC, Mealey BL, van Dyke TE y cols.Consensus report: Periodontal health and gingival diseases/conditions. Journal of Clinical Periodontology. 2018; 45: p. S68-S77.

8 Holmstrup P, Plemons J, Meyle J. Non-plaque induced gingival diseases. Journal of Clinical Periodontology. 2018; 45: p. $S 28-S 43$.

9 Stone SJ, Heasman PA, Staines KS, McCracken GI. The impact of structured plaque control for patients with gingival manifestations of oral lichen planus: A randomized controlled study. Journal of Clinical Periodontology. 2015; 42: p. 356-362.

10 Kumar DG, Kundapur PP, Bhat GS, Bhat KM, Guddattu V. Periodontal disease and obesity in an Indian population. Nepal Journal of Medical Sciences. 2013 Jul; 2(2): p. 144148.

11 Al-Qahtani SM, Elagib MF, Reddy NR, Alghamdi NS, Baldo SM, Kumar MP. Relationship between Obesity and Periodontal Diseases in Saudi Women (Asir Region): A Prospective Study. The Journal of Contemporary Dental Practice. 2018 Aug; 19(8): p. 969-973.

12 Merello, LM, Oliva MP. La Obesidad y su Relación con las Patologías Periodontales: una Revisión Narrativa. Int. J. Odontostomat. 2012 Oct; 6(2): p. 235-239.

13 Martens L, De Smet S, Yusof MY, Rajasekharan S. : Association between overweight/obesity and periodontal disease 
in children and adolescents: a systematic review and metaanalysis. European Academy of Paediatric Dentistry. 2017 Nov; 18: p. 69-82.

14 Ministerio de Salud Pública del Ecuador. Manual de Uso de los Formularios Básicos de la Historia Clínica Única, 2010; p. 1-38.

15 Ansari S. The Relationship between Body Mass Index and Periodontal Disease. Scholars Journal of Applied Medical Sciences. 2019 Enero; 7(2): p. 512-516.

16 Muniz F, Costa R, Cavagni J, Milanesi F, Hilgert J, Gomes S, Oppermann R, Rösing C. Body fat rather than body mass index is associated with gingivitis - A southern Brazilian cross-sectional study. J Periodontol. 2018 Apr;89(4):388396.

17 Zuza A, BarrosoEM, Rosetti EP, Pires J. Periodontal Disease and Body Weight Assessment in Children. Journal of Dentistry for Children. 2017; 84:1-8.

18 Martinez M, Silvestre J y Silvestre F. Association between obesity and periodontal disease. A systematic review of epidemiological studies and controlled clinical trials. Med Oral Patol Oral Cir Bucal. 2017 Nov; 22(6): e708-e715.

19 Pérez MM, Pessoa JS, Guaré RO. Correlation of salivary immunoglobulin A with Body Mass Index and fat percentage in overweight/obese children. Journal of applied oral science. 2018 Junio; 27(20): p. 1-8.

20 Chopra A, Lakhanpal M, Gupta N, Rao NC. Association between obesity and periodontal disease: A cross-sectional study. Saudi J Obesity. 2013; 1(2): p. 71.

21 Franchini R, Petri A, Migliario M, Rimondini L. Poor oral hygiene and gingivitis are associated with obesity and overweight status in paediatric subjects. Journal Of Clinical Periodontology. 2011 Nov;38(11):p. 1021-8

22 Fadel H, Pliaki A, Gronowitz E, Mårild S, Ramberg P, Dahlèn G, Yucel-Lindberg T, Heijl L, Birkhed D. Clinical and biological indicators of dental caries and periodontal disease in adolescents with or without obesity. Clin Oral Investig. 2014; 18(2): p. 359-368.

23 Juarez I, Hernández M, Letelier C, Halabí D, Araneda C. Association between abdominal obesity and periodontal disease. Cross-sectional study. J Oral Rescarch.2015; 4(2):95-102.

Recibido: 01 de Marzo del 2020

Aceptado: 6 de Abril del 2020 